\title{
Research Article \\ Stability Analysis of Recurrent Neural Networks with Random Delay and Markovian Switching
}

\author{
Enwen Zhu, ${ }^{1,2}$ Yong Wang, ${ }^{3}$ Yueheng Wang, ${ }^{1}$ Hanjun Zhang, ${ }^{2}$ \\ and Jiezhong Zou ${ }^{4}$ \\ ${ }^{1}$ School of Mathematics and Computational Science, Changsha University of Science and Technology, \\ 410076 Hunan, China \\ 2 School of Mathematics and Computational Science, Xiangtan University, 411105 Hunan, China \\ ${ }^{3}$ Department of Mathematics, Harbin Institute of Technology, 150001 Heilongjiang, China \\ ${ }^{4}$ School of Mathematics, Central South University, 410075 Hunan, China
}

Correspondence should be addressed to Enwen Zhu, engwenzhu@126.com

Received 14 March 2010; Accepted 18 May 2010

Academic Editor: Alexander I. Domoshnitsky

Copyright ( 2010 Enwen Zhu et al. This is an open access article distributed under the Creative Commons Attribution License, which permits unrestricted use, distribution, and reproduction in any medium, provided the original work is properly cited.

\begin{abstract}
In this paper, the exponential stability analysis problem is considered for a class of recurrent neural networks (RNNs) with random delay and Markovian switching. The evolution of the delay is modeled by a continuous-time homogeneous Markov process with a finite number of states. The main purpose of this paper is to establish easily verifiable conditions under which the random delayed recurrent neural network with Markovian switching is exponentially stable. The analysis is based on the Lyapunov-Krasovskii functional and stochastic analysis approach, and the conditions are expressed in terms of linear matrix inequalities, which can be readily checked by using some standard numerical packages such as the Matlab LMI Toolbox. A numerical example is exploited to show the usefulness of the derived LMI-based stability conditions.
\end{abstract}

\section{Introduction}

In recent years, the neural networks (NNs) have been extensively studied because of their immense application potentials, such as signal processing, pattern recognition, static image processing, associative memory, and combinatorial optimization. In practice, time delays are frequently encountered in dynamical systems and are often a source of oscillation and instability. Thus, the stability problem of delayed neural networks has become a topic of great theoretic and practical importance. Numerous important results have been reported for neural networks with time delays (see, e.g., [1-23]). 
On the other hand, it can be seen from the existing references that only the deterministic time-delay case was concerned, and the stability criteria were derived based only on the information of variation range of the time delay. In practice, the delay in some NNs is due to multiple factors (e.g., synaptic transmission delay, neuroaxon transmission delay); one natural paradigm for treating it is to use a probabilistic description (see, e.g., [17, 18, 24, 25]). For example, to control and propagate the stochastic signals through universal learning networks (ULNs), a probabilistic universal learning network (PULN) was proposed in [25]. In a PULN, the output signal of the node is transferred to another node by multibranches with arbitrary time delay which is random and its probabilistic characteristic can often be measured by the statistical methods. For this case, if some values of the time delay are very large but the probabilities of the delay taking such large values are very small, it may result in a more conservative result if only the information of variation range of the time delay is considered. In many situations, the delay process can be modeled as a Markov process with a finite number of states (see, e.g., [26, 27]). References [26, 27] argue in favor of such representation of the delay in communication networks. The discrete values of the delay may correspond to "low", "medium", and "high" network loads.

In practice, sometimes a neural network has finite state representations (also called modes, patterns, or clusters), and the modes may switch (or jump) from one to another at different times [19-23]. Recently, it has been revealed in [19] that switching (or jumping) between different neural networks modes can be governed by a Markov chain. Specifically, the class of neural networks with Markovian switching has two components in the state vector. The first one, which carries continuously, is referred to be the continuous state of the neural networks, and the second one, which varies discretely, is referred to be the mode of the neural networks. For a specific mode, the dynamics of the neural networks is continuous, but the switchings among different modes may be seen as discrete events. It should be pointed out that neural networks with Markovian switching have been a subject of great significance in modeling a class of neural networks with finite network modes and were studied by several researchers, for example, [19-23, 28], despite their practical importance. However, to the best of the authors' knowledge, so far, the stability analysis of RNNs with random delay and Markovian switching has received little attention in the literature. This situation motivates our present investigation.

Motivated by the above discussions, the aim of this paper is to investigate the exponential stability of RNNs with random delay and Markovian switching in mean square. By using a Markov chain with a finite number of states, we propose a new model of RNNs with random delay and Markovian switching. The analysis is based on the LyapunovKrasovskii functional and stochastic analysis approach, and the conditions for the stability criteria are expressed in terms of linear matrix inequalities which can be readily checked by using some standard numerical packages. A simple example has been provided to demonstrate the effectiveness and applicability of the proposed testing criteria.

\section{Notations}

The notations are quite standard. Throughout this paper, $\mathbb{R}^{n}$ and $\mathbb{R}^{n \times m}$ denote, respectively, the $n$-dimensional Euclidean space and the set of all $n \times m$ real matrices. The superscript " $T$ " denotes the transpose and the notation $X \geq Y$ (resp., $X>Y$ ), where $X$ and $Y$ are symmetric matrices, means that $X-Y$ is positive semidefinite (resp., positive definite). $I$ is the identity matrix with compatible dimension. For $h>0, C\left([-h, 0] ; \mathbb{R}^{n}\right)$ denotes the family of continuous functions $\varphi$ from $[-h, 0]$ to $\mathbb{R}^{n}$ with the norm $\|\varphi\|=\sup _{-h \leq \theta \leq 0}|\varphi(\theta)|$, where $|\cdot|$ is the Euclidean 
norm in $\mathbb{R}^{n}$. If $A$ is a matrix, denote by $\|A\|$ its operator norm, that is, $\|A\|=\sup \{|A x|$ : $|x|=1\}=\sqrt{\lambda_{\max }\left(A^{T} A\right)}$, where $\lambda_{\max }(\cdot)$ (resp., $\left.\lambda_{\min }(\cdot)\right)$ means the largest (resp., smallest) eigenvalue of $A$. Moreover, let $\left(\Omega, \mathcal{F},\left\{\mathcal{F}_{t}\right\}_{t \geq 0}, \mathrm{P}\right)$ be complete probability space with a filtration $\left\{\mathscr{F}_{t}\right\}_{t>0}$ (i.e., it is right continuous and $\mathcal{F}_{0}$ contains all P-null sets). Denote by $C_{\Phi_{0}}^{b}\left([-h, 0] ; \mathbb{R}^{n}\right)$ the family of all bounded, $\mathcal{F}_{0}$-measurable, $C\left([-h, 0] ; \mathbb{R}^{n}\right)$-valued random variables. For $p>0$ and $h>0$, denote by $L_{千_{0}}^{p}\left([-h, 0] ; \mathbb{R}^{n}\right)$ the family of all $\mp_{0}$-measurable, $C\left([-h, 0] ; \mathbb{R}^{n}\right)$-valued random variables $\phi=\{\phi(\theta):-h \leq \theta \leq 0\}$ such that $\sup _{-h \leq \theta \leq 0} \mathbb{E}|\phi(\theta)|^{p}<\infty$, where $\mathbb{E}$ stands for the mathematical expectation operator with respect to the given probability measure $\mathrm{P}$. In symmetric block matrices, we use an asterisk " $*$ " to represent a term that is induced by symmetry and $\operatorname{diag}\{\cdots\}$ stands for a block-diagonal matrix. Sometimes, the arguments of a function will be omitted in the analysis when no confusion can arise.

\section{Problem Formulation}

In this section, we will introduce the model of recurrent neural networks with random delay and Markovian switching, give the definition of stability related, and put forward the problem to be dealt with in this paper.

Let $\{\eta(t), t \geq 0\}$ be a right-continuous Markov process on the probability space which takes values in the finite space $S=\left\{\eta_{1}, \eta_{2}, \ldots, \eta_{N}\right\}$ satisfying $0 \leq \eta_{1}, \eta_{2}, \ldots, \eta_{N} \leq h$ and its generator $\Gamma=\left(\gamma_{i j}\right)_{N \times N}$ is given by

$$
\mathrm{P}\left\{\eta(t+\Delta)=\eta_{j} \mid \eta(t)=\eta_{i}\right\}= \begin{cases}\gamma_{i j} \Delta+o(\Delta) & \text { if } \eta_{i} \neq \eta_{j}, \\ 1+\gamma_{i i}+o(\Delta) & \text { if } \eta_{i}=\eta_{j},\end{cases}
$$

where $\Delta>0$ and $\lim _{\Delta \rightarrow 0} O(\Delta) / \Delta=0, \gamma_{i j} \geq 0$ is the transition rate from $\eta_{i}$ to $\eta_{j}$ if $\eta_{i} \neq \eta_{j}$, and

$$
\gamma_{i i}=-\sum_{\eta_{j} \neq \eta_{i}} \gamma_{i j}
$$

Consider the following recurrent neural network with constant delay model described by

$$
\dot{u}(t)=-A u(t)+W_{0} f_{0}(u(t))+W_{1} f_{1}(u(t-h))+V,
$$

where $u(t)=\left(u_{1}(t), u_{2}(t), \ldots, u_{n}(t)\right)^{T} \in \mathbb{R}^{n}$ is the states vector with the $n$ neurons; the diagonal matrix $A=\operatorname{diag}\left(a_{1}, a_{2}, \ldots, a_{n}\right)$ has positive entries $a_{i}>0 ; W_{0}=\left(W_{i j}^{0}\right)_{n \times n} \in \mathbb{R}^{n \times n}$ and $W_{1}=$ $\left(W_{i j}^{1}\right)_{n \times n} \in \mathbb{R}^{n \times n}$ are the connection weight matrix and delayed connection weight matrix, respectively; $f_{k}(u(t))=\left(f_{k 1}\left(u_{1}(t)\right), \ldots, f_{k n}\left(u_{n}(t)\right)\right)^{T}(k=0,1)$ denotes the neuron activation function with $f_{k}(0)=0 ; V=\left[V_{1}, V_{2}, \ldots, V_{n}\right]^{T}$ is a constant external input vector; $h>0$, which may be unknown, denotes the time delay.

Throughout this paper, we make the following assumptions. 
Assumption 2.1. For $i \in\{1,2, \ldots, n\}$, the neuron activation functions in (2.3) satisfy

$$
\begin{aligned}
& 0 \leq \frac{f_{0 i}(x)-f_{0 i}(y)}{x-y} \leq k_{0}, \\
& 0 \leq \frac{f_{1 i}(x)-f_{1 i}(y)}{x-y} \leq k_{1},
\end{aligned}
$$

for any $x, y \in \mathbb{R}, x \neq y$, where $k_{0}$ and $k_{1}$ are positive constants.

Remark 2.2. It is obvious that the conditions in Assumption 2.1 are more general than the usual sigmoid functions and the recently commonly used Lipschitz conditions; see, for example, [3-7].

Let $u^{*}=\left(u_{1}^{*}, u_{2}^{*}, \ldots, u_{n}^{*}\right)$ be the equilibrium point of network (2.3). For the purpose of simplicity, we can shift the intended equilibrium points $u^{*}$ to the origin. The transformation, that is, $x(t)=u(t)-u^{*}$, puts system (2.3) into the following form:

$$
\dot{x}(t)=-A x(t)+W_{0} g_{0}(x(t))+W_{1} g_{1}(x(t-h)),
$$

where $x(t)=\left(x_{1}(t), x_{2}(t), \ldots, x_{n}(t)\right)^{T} \in \mathbb{R}^{n}$ is the state vector of transformed system, $g_{k}(x(\cdot))=\left(g_{k 1}\left(x_{k 1}(\cdot)\right), g_{k 2}\left(x_{k 2}(\cdot)\right), \ldots, g_{k n}\left(x_{k n}(\cdot)\right)\right)^{T}$, and $g_{k}(x(\cdot))=f_{k}\left(x(\cdot)+u^{*}\right)-f_{k}\left(u^{*}\right)(k=$ $0,1)$. Obviously, Assumption 2.1 implies that $g_{k i}(\cdot)(k=0,1)$ satisfy the following condition:

$$
\begin{aligned}
& 0 \leq \frac{g_{0 i}\left(x_{i}\right)}{x_{i}} \leq k_{0}, \quad \forall x_{i} \neq 0, i=1,2, \ldots, n, \\
& 0 \leq \frac{g_{1 i}\left(x_{i}\right)}{x_{i}} \leq k_{1}, \quad \forall x_{i} \neq 0, i=1,2, \ldots, n,
\end{aligned}
$$

and from (2.7), we have

$$
\begin{array}{ll}
g_{0 i}\left(x_{i}\right)\left(g_{0 i}\left(x_{i}\right)-k_{0} x_{i}\right) \leq 0, & i=1,2, \ldots, n \\
g_{1 i}\left(x_{i}\right)\left(g_{1 i}\left(x_{i}\right)-k_{1} x_{i}\right) \leq 0, & i=1,2, \ldots, n
\end{array}
$$

Now we consider the following recurrent neural network with random delay and Markovian switching, which is actually a modification of (2.6):

$$
\dot{x}(t)=-A(\eta(t)) x(t)+W_{0}(\eta(t)) g_{0}(x(t))+W_{1}(\eta(t)) g_{1}(x(t-\eta(t))),
$$

where $\eta(t)$ is a Markov process taking values in a finite state space $S=\left\{\eta_{1}, \eta_{2}, \ldots, \eta_{N}\right\}$. 
Assumption 2.3. The neuron activation function in (2.9), $g_{1}$, satisfies the following condition:

$$
g_{1 j}\left(x_{1 j}\left(t-\eta_{i}\right)\right) x_{1 j}\left(t-\eta_{i}\right) \leq \rho\left(x_{1 j}\left(t-\eta_{i}\right)\right)^{2}, \quad j=1,2, \ldots, n, 0 \leq \eta_{i} \leq h,
$$

where $\rho$ is a positive constant.

Now we will work on the network mode $\eta(t)=\eta_{i}$, for all $\eta_{i} \in S$. Let $x(t ; \xi)$ denote the state trajectory from the initial data $x(\theta)=\xi(\theta)$ on $-h \leq \theta \leq 0$ in $L_{\mathscr{f}_{0}}^{2}\left([-h, 0] ; \mathbb{R}^{n}\right)$. According to $[26,29]$, for any initial value $\xi,(2.9)$ has only a globally continuous state. Clearly, the network (2.9) admits an equilibrium point (trivial solution) $x(t ; 0) \equiv 0$ corresponding to the initial data $\xi=0$.

Remark 2.4. It is noted that the introduction of random delay modeled by a continuous-time homogeneous Markov process with a finite number of states was first introduced in [26,27]. Unlike the common assumptions on the delay in the published literature, the probability distribution of the delay taking some values is assumed to be known in advance in this paper, and then a new model of the neural system (2.9) has been derived, which can be seen as an extension of the common neural system (2.6).

For convenience, each possible value of $\eta(t)$ is denoted by $\eta_{i}, \eta_{i} \in S$ in the sequel. Then we have

$$
A_{i}:=A\left(\eta(t)=\eta_{i}\right), \quad W_{0 i}:=W_{0}\left(\eta(t)=\eta_{i}\right), \quad W_{1 i}:=W_{1}\left(\eta(t)=\eta_{i}\right),
$$

where $A_{i}, W_{0 i}, W_{1 i}$ for any $\eta_{i} \in S$ are known constant matrices of appropriate dimensions. The following stability concept is needed in this paper.

Definition 2.5. For system (2.9) and every $\xi \in L_{\mp_{0}}^{2}\left([-h, 0], \mathbb{R}^{n}\right), \eta(0)=\eta_{i_{0}} \in S$, the trivial solution is exponentially stable in the mean-square if there exists a pair of positive constants $\alpha$ and $\beta$ such that

$$
\mathbb{E}\left|x\left(t, \xi, \eta_{i_{0}}\right)\right|^{2} \leq \alpha e^{-\beta t} \sup _{-h \leq \theta \leq 0} \mathbb{E}|\xi(\theta)|^{2}, \quad t \geq 0,
$$

where $x\left(t, \xi, \eta_{i_{0}}\right)$ is the solution of system (2.9) at time $t$ under the initial state $\xi$ and initial mode $\eta_{i_{0}}$.

\section{Main Results and Proofs}

To establish a more general result, we need more notations. Let $C^{2,1}\left(\mathbb{R}^{n} \times \mathbb{R}_{+} \times S ; \mathbb{R}_{+}\right)$denote the family of all nonnegative functions $V\left(x, t, \eta_{i}\right)$ on $\mathbb{R}^{n} \times \mathbb{R}_{+} \times S$, which are continuously twice 
differentiable in $x$ and differentiable in $t$. If $V \in C^{2,1}\left(\mathbb{R}^{n} \times \mathbb{R}_{+} \times S ; \mathbb{R}_{+}\right)$, define an operator $\mathcal{L} V$ from $\mathbb{R}^{n} \times \mathbb{R}_{+} \times S$ to $\mathbb{R}$ by

$$
\begin{aligned}
\mathcal{L}\left(x(t), t, \eta_{i}\right)= & V_{t}\left(x(t), t, \eta_{i}\right)+V_{x}\left(x(t), t, \eta_{i}\right) \\
& \times\left[-A_{i} x(t)+W_{0 i} g_{0}(x(t))+W_{1 i} g_{1}\left(x\left(t-\eta_{i}\right)\right)\right] \\
& +\sum_{j=1}^{N} \gamma_{i j} V\left(x(t), t, \eta_{j}\right)
\end{aligned}
$$

where

$$
\begin{gathered}
V_{t}\left(x, t, \eta_{i}\right)=\frac{\partial V\left(x, t, \eta_{i}\right)}{\partial t} \\
V_{x}\left(x, t, \eta_{i}\right)=\left(\frac{\partial V\left(x, t, \eta_{i}\right)}{\partial x_{1}}, \ldots, \frac{\partial V\left(x, t, \eta_{i}\right)}{\partial x_{n}}\right) .
\end{gathered}
$$

The main result of this paper is given in the following theorem.

Theorem 3.1. Let $\epsilon$ be a fixed constant. Then under Assumptions 2.1 and 2.3, the recurrent neural network (2.9) with random delay and Markovian switching is exponentially stable in the mean square if there exist symmetric positive matrices $P_{i}, Q$ and positive diagonal matrices $D_{i}=$ $\operatorname{diag}\left(d_{1 i}, d_{2 i}, \ldots, d_{n i}\right), \quad R_{i}, \Lambda=\operatorname{diag}\left(\lambda_{1}, \lambda_{2}, \ldots, \lambda_{n}\right)$ such that the following LMIs holds

$$
\begin{gathered}
M_{1}:=\left[\begin{array}{ccc}
(1.1) & P_{i} W_{0 i}-A_{i} D_{i} & P_{i} W_{1 i} \\
* & -R_{i}+D_{i} W_{0 i}+W_{0 i}^{T} D_{i} & D_{i} W_{1 i} \\
* & * & -2 \Lambda
\end{array}\right]<0, \\
\quad-e^{-2 \epsilon \eta_{i}} Q+2 k_{1} \rho \Lambda \leq 0, \\
(1.1)=2 \epsilon P_{i}+2 \epsilon k_{0} D_{i}+k_{0}^{2} R_{i}-\left(P_{i} A_{i}+A_{i} P_{i}\right)+\sum_{j=1}^{N} \gamma_{i j} P_{j} .
\end{gathered}
$$

Proof. In order to establish the stability conditions, we define a Lyapunov functional candidate $V\left(x(t), t, \eta(t)=\eta_{i}\right):=V\left(x(t), t, \eta_{i}\right) \in C^{2,1}\left(\mathbb{R}^{n} \times \mathbb{R}_{+} \times S ; \mathbb{R}_{+}\right)$by

$$
\begin{aligned}
V\left(x(t), t, \eta(t)=\eta_{i}\right)= & e^{2 \epsilon t} x^{T}(t) P_{i} x(t)+2 e^{2 \epsilon t} \sum_{k=1}^{n} d_{k i} \int_{0}^{x_{k}(t)} g_{0}(s) d s \\
& +\int_{t-\eta(t)}^{t} e^{2 \epsilon s} x^{T}(s) Q x(s) d s .
\end{aligned}
$$


It is known (see $[26,29])$ that $\{x(t), \eta(t)\}$ is a $C\left([-h, 0] ; \mathbb{R}^{n}\right) \times S$-valued Markov process. From (2.9), (3.1), and (3.4), the weak infinitesimal operator $\mathcal{L}$ (see [30]) of the stochastic process $\{x(t), \eta(t)\}$ is given by

$$
\begin{aligned}
\mathcal{L} V( & \left.x(t), t, \eta(t)=\eta_{i}\right): \\
= & \lim _{\Delta \rightarrow 0^{+}} \frac{1}{\Delta}\left[\mathbb{E}\left\{V(x(t+\Delta), \eta(t+\Delta)) \mid x(t), \eta(t)=\eta_{i}\right\}-V\left(x(t), \eta(t)=\eta_{i}\right)\right] \\
= & 2 \epsilon e^{2 \epsilon t} x^{T}(t) P_{i} x(t)+4 \epsilon e^{2 \epsilon t} \sum_{k=1}^{n} d_{k i} \int_{0}^{x_{k}(t)} g_{0}(s) d s \\
& +2 e^{2 \epsilon t} x^{T}(t) P_{i}\left[-A_{i} x(t)+W_{0 i} g_{0}(x(t))+W_{1 i} g_{1}\left(x\left(t-\eta_{i}\right)\right)\right] \\
& +2 e^{2 \epsilon t} g_{0}^{T}(x(t)) D_{i}\left[-A_{i} x(t)+W_{0 i} g_{0}(x(t))+W_{1 i} g_{1}\left(x\left(t-\eta_{i}\right)\right)\right] \\
& +e^{2 \epsilon t} x^{T}(t) Q x(t)+e^{2 \epsilon t} \sum_{j=1}^{N} \gamma_{i j} x^{T}(t) P_{j} x(t) \\
& -e^{2 \epsilon\left(t-\eta_{i}\right)} x^{T}\left(t-\eta_{i}\right) Q x\left(t-\eta_{i}\right) .
\end{aligned}
$$

By using (2.7), we have

$$
\int_{0}^{x_{k}(t)} g_{0}(s) d s \leq \frac{k_{0}}{2} x_{k}^{2}(t)
$$

It is easy to see that

$$
\sum_{k=1}^{n} d_{k i} \int_{0}^{x_{k}(t)} g_{0}(s) d s \leq \frac{k_{0}}{2} x^{T}(t) D_{i} x(t) .
$$

Substituting (2.8), (2.10), and (3.7) into (3.5) leads to

$$
\begin{aligned}
\mathcal{L} V\left(x(t), t, \eta(t)=\eta_{i}\right) \leq e^{2 \epsilon t}\{ & 2 \epsilon x^{T}(t) P_{i} x(t)+g_{0}^{T}(x(t)) R_{i} g_{0}(x(t))-g_{0}^{T}(x(t)) R_{i} g_{0}(x(t)) \\
& -2 x^{T}(t) P_{i} A_{i} x(t)+2 x^{T}(t) P_{i} W_{0 i} g_{0}(x(t)) \\
& +2 x^{T}(t) P_{i} W_{1 i} g_{1}\left(x\left(t-\eta_{i}\right)\right) \\
& +2 \epsilon x^{T}(t) k_{0} D_{i} x(t)-2 g_{0}^{T}(x(t)) D_{i} A_{i} x(t) \\
& +2 g_{0}^{T}(x(t)) D_{i} W_{0 i} g_{0}(x(t)) \\
& +2 g_{0}^{T}(x(t)) D_{i} W_{1 i} g_{1}\left(x\left(t-\eta_{i}\right)\right) \\
& \left.+\sum_{j=1}^{N} \gamma_{i j} x^{T}(t) P_{j} x(t)+x^{T}(t) Q x(t)-e^{-2 \epsilon \eta_{i}} x^{T}\left(t-\eta_{i}\right) Q x\left(t-\eta_{i}\right)\right\}
\end{aligned}
$$




$$
\begin{aligned}
& \leq e^{2 \epsilon t}\left\{2 \epsilon x^{T}(t) P_{i} x(t)+g_{0}^{T}(x(t)) R_{i} g_{0}(x(t))-g_{0}^{T}(x(t)) R_{i} g_{0}(x(t))\right. \\
& -2 x^{T}(t) P_{i} A_{i} x(t)+2 x^{T}(t) P_{i} W_{0 i} g_{0}(x(t)) \\
& +2 x^{T}(t) \mathrm{P}_{i} W_{1 i} g_{1}\left(x\left(t-\eta_{i}\right)\right) \\
& +2 \epsilon x^{T}(t) k_{0} D_{i} x(t)-2 g_{0}^{T}(x(t)) D_{i} A_{i} x(t) \\
& +2 g_{0}^{T}(x(t)) D_{i} W_{0 i} g_{0}(x(t)) \\
& +2 g_{0}^{T}(x(t)) D_{i} W_{1 i} g_{1}\left(x\left(t-\eta_{i}\right)\right)+\sum_{j=1}^{N} \gamma_{i j} x^{T}(t) P_{j} x(t) \\
& +x^{T}(t) Q x(t)-e^{-2 \epsilon \eta_{i}} x^{T}\left(t-\eta_{i}\right) Q x\left(t-\eta_{i}\right) \\
& \left.-2 \sum_{j} \lambda_{j} g_{1}\left(x_{j}\left(t-\eta_{i}\right)\right)\left(g_{1}\left(x_{j}\left(t-\eta_{i}\right)\right)-k_{1} x_{j}\left(t-\eta_{i}\right)\right)\right\} \\
& \leq e^{2 \epsilon t}\left\{\left[\begin{array}{ll}
x^{T}(t) & g_{0}^{T}(x(t)) g_{1}^{T}\left(x\left(t-\eta_{i}\right)\right)
\end{array}\right] M_{1}\left[\begin{array}{c}
x(t) \\
g_{0}(x(t)) \\
g_{1}\left(x\left(t-\eta_{i}\right)\right)
\end{array}\right]\right. \\
& \left.+x^{T}\left(t-\eta_{i}\right)\left(-e^{-2 \epsilon \eta_{i}} Q+2 k_{1} \rho \Lambda\right) x\left(t-\eta_{i}\right)\right\} \text {. }
\end{aligned}
$$

Using the conditions of Theorem 3.1, we have

$$
\mathcal{L V}\left(x(t), t, \eta(t)=\eta_{i}\right)<0 .
$$

Hence

$$
\mathbb{E} V\left(x(t), t, \eta(t)=\eta_{i}\right) \leq \mathbb{E} V(x(0), 0, \eta(0)),
$$

where

$$
\begin{aligned}
\mathbb{E} V(x(0), 0, \eta(0)) & =\mathbb{E}\left\{x^{T}(0) P_{i} x(0)+2 \sum_{k=1}^{n} d_{k i} \int_{0}^{x_{k}(t)} g_{0}(s) d s+\int_{-\eta(0)}^{0} e^{2 \epsilon s} x^{T}(s) Q x(s) d s\right\} \\
& \leq\left\{\lambda_{\max }\left(P_{i}\right)+2 k_{0} \max _{1 \leq k \leq n} d_{k i}+\lambda_{\max }(Q) \frac{1-e^{-2 \epsilon \eta(0)}}{2 \epsilon}\right\} \mathbb{E}\|\xi\|^{2} .
\end{aligned}
$$


Moreover

$$
\mathbb{E} V\left(x(t), t, \eta(t)=\eta_{i}\right) \geq \lambda_{\min }\left(P_{i}\right) e^{2 \epsilon t} \mathbb{E}|x(t)|^{2} .
$$

Combining (3.10)-(3.12), we have

$$
\begin{aligned}
\lambda_{\min }\left(P_{i}\right) e^{2 \epsilon t} \mathbb{E}|x(t)|^{2} & \leq \mathbb{E} V\left(x(t), t, \eta(t)=\eta_{i}\right) \\
& \leq\left\{\lambda_{\max }\left(P_{i}\right)+2 k_{0} \max _{1 \leq k \leq n} d_{k i}+\lambda_{\max }(Q) \frac{1-e^{-2 \epsilon \eta(0)}}{2 \epsilon}\right\} \mathbb{E}\|\xi\|^{2} .
\end{aligned}
$$

Hence

$$
\mathbb{E}|x(t)|^{2} \leq \alpha e^{-2 \epsilon t} \mathbb{E}\|\xi\|^{2}
$$

where

$$
\alpha=\frac{\lambda_{\max }\left(P_{i}\right)+2 k_{0} \max _{1 \leq k \leq n} d_{k i}+\lambda_{\max }(Q)\left(\left(1-e^{-2 \epsilon \eta(0)}\right) / 2 \epsilon\right)}{\lambda_{\min }\left(P_{i}\right)},
$$

which implies that system (2.9) is exponentially stable in the mean square sense. The proof is completed.

\section{A Numerical Example}

In this section, a numerical example is presented to demonstrate the effectiveness and applicability of the developed method on the exponential stability in the mean square sense of the recurrent neural network (2.9) with random delay and Markovian switching.

Example 4.1. Consider a two-neuron neural network (2.9) with two modes. The network parameters are given as follows:

$$
\begin{gathered}
A_{1}=\left[\begin{array}{cc}
1.6 & 0 \\
0 & 1.8
\end{array}\right], \quad A_{2}=\left[\begin{array}{cc}
2.2 & 0 \\
0 & 1.5
\end{array}\right], \quad W_{01}=\left[\begin{array}{cc}
1.2 & -1.5 \\
-1.7 & 1.2
\end{array}\right], \\
W_{02}=\left[\begin{array}{cc}
-0.6 & 0.1 \\
0.1 & 0.2
\end{array}\right], \quad W_{11}=\left[\begin{array}{cc}
-1.1 & 0.5 \\
0.5 & 0.8
\end{array}\right], \quad W_{12}=\left[\begin{array}{cc}
0.8 & 0.2 \\
0.2 & 0.3
\end{array}\right], \\
\Gamma=\left[\begin{array}{cc}
-6 & 6 \\
1 & -1
\end{array}\right], \quad k_{0}=k_{1}=1, \epsilon=0.3, \rho=1.5, \eta_{1}=0.5, \eta_{2}=0.3 .
\end{gathered}
$$


By using the Matlab LMI toolbox [31], we solve the LMIs in Theorem 3.1 and obtain

$$
\begin{array}{cc}
P_{1}=\left[\begin{array}{cc}
41.7575 & 3.5729 \\
3.5729 & 39.7220
\end{array}\right]>0, & P_{2}=\left[\begin{array}{cc}
22.6299 & 8.5934 \\
8.5934 & 24.7458
\end{array}\right]>0, \\
D_{1}=\left[\begin{array}{cc}
1.7450 & 0 \\
0 & 1.7300
\end{array}\right]>0, & D_{2}=\left[\begin{array}{cc}
1.5125 & 0 \\
0 & 3.7358
\end{array}\right]>0, \\
Q=\left[\begin{array}{cc}
276.3824 & 0 \\
0 & 194.9230
\end{array}\right]>0, & \Lambda=\left[\begin{array}{cc}
50.0866 & 0 \\
0 & 34.2662
\end{array}\right]>0, \\
R_{1}=\left[\begin{array}{cc}
106.8949 & 0 \\
0 & 96.6521
\end{array}\right]>0, & R_{2}=\left[\begin{array}{cc}
16.7054 & 0 \\
0 & 12.5835
\end{array}\right]>0 .
\end{array}
$$

Therefore, it follows from Theorem 3.1 that the recurrent neural network (2.9) with random delay and Markovian switching is exponentially stable in the mean square.

\section{Conclusions}

The analysis problem of an exponential stability in the mean square sense for a class of RNNs with random delay and Markovian switching has been studied. By utilizing a Markov chain to describe discrete delay, a new neural network model has been presented. The LyapunovKrasovskii stability theory and the differential rule have been employed to establish sufficient conditions for the recurrent neural network with random delay and Markovian switching to be exponentially stable. These conditions are expressed in terms of the feasibility to a set of linear matrix inequalities, and therefore the exponentially stable of the recurrent neural network with random delay and Markovian switching can be easily checked by utilizing the numerically efficient Matlab LMI toolbox. A simple example has been exploited to show the usefulness of the derived LMI-based stability conditions.

\section{Acknowledgments}

The authors would like to thank the editor and the anonymous referees for their detailed comments and valuable suggestions which considerably improved the presentation of this paper. The authors would like to thank Prof. Fuzhou Gong for his guidance. The research is supported by the Excellent Youth Foundation of Educational Committee of Hunan Provincial (08B005), the Hunan Postdoctoral Scientific Program (2009RS3020), the National Natural Science Foundation of China (10771044), the Natural Science Foundation of Hunan Province (09JJ6006), the Scientific Research Funds of Hunan Provincial Education Department of China (09C059), and the Scientific Research Funds of Hunan Provincial Science and Technology Department of China (2009FJ3103). 


\section{References}

[1] S. Arik, "Stability analysis of delayed neural networks," IEEE Transactions on Circuits and Systems I, vol. 47, no. 7, pp. 1089-1092, 2000.

[2] P. P. Civalleri, M. Gilli, and L. Pandolfi, “On stability of cellular neural networks with delay," IEEE Transactions on Circuits and Systems I, vol. 40, no. 3, pp. 157-165, 1993.

[3] J. Cao, "Global exponential stability and periodic solutions of delayed cellular neural networks," Journal of Computer and System Sciences, vol. 60, no. 1, pp. 38-46, 2000.

[4] H. Zhao, "Global exponential stability and periodicity of cellular neural networks with variable delays," Physics Letters A, vol. 336, no. 4-5, pp. 331-341, 2005.

[5] Z. Wang, Y. Liu, and X. Liu, "On global asymptotic stability of neural networks with discrete and distributed delays," Physics Letters A, vol. 345, no. 4-6, pp. 299-308, 2005.

[6] T. Chen, W. Lu, and G. Chen, "Dynamical behaviors of a large class of general delayed neural networks," Neural Computation, vol. 17, no. 4, pp. 949-968, 2005.

[7] X. Y. Lou and B. Cui, "New LMI conditions for delay-dependent asymptotic stability of delayed Hopfield neural networks," Neurocomputing, vol. 69, no. 16-18, pp. 2374-2378, 2006.

[8] Y. He, G. Liu, and D. Rees, "New delay-dependent stability criteria for neural networks with timevarying delay," IEEE Transactions on Neural Networks, vol. 18, pp. 310-314, 2007.

[9] C. Zheng, L. Lu, and Z. Wang, "New LMI-based delay-dependent criterion for global asymptotic stability of cellular neural networks," Neurocomputing, vol. 72, pp. 3331-3336, 2009.

[10] X. X. Liao and X. Mao, "Stability of stochastic neural networks," Neural, Parallel E Scientific Computations, vol. 4, no. 2, pp. 205-224, 1996.

[11] X. X. Liao and X. Mao, "Exponential stability and instability of stochastic neural networks," Stochastic Analysis and Applications, vol. 14, no. 2, pp. 165-185, 1996.

[12] S. Blythe, X. Mao, and X. Liao, "Stability of stochastic delay neural networks," Journal of the Franklin Institute, vol. 338, no. 4, pp. 481-495, 2001.

[13] L. Wan and J. Sun, "Mean square exponential stability of stochastic delayed Hopfield neural networks," Physics Letters A, vol. 343, no. 4, pp. 306-318, 2005.

[14] Y. Sun and J. Cao, " $p$ th moment exponential stability of stochastic recurrent neural networks with time-varying delays," Nonlinear Analysis: Real World Applications, vol. 8, no. 4, pp. 1171-1185, 2007.

[15] L. Ma and F. Da, "Mean-square exponential stability of stochastic Hopfield neural networks with time-varying discrete and distributed delays," Physics Letters A, vol. 373, no. 25, pp. 2154-2161, 2009.

[16] Y. Zhang, D. Yue, and E. Tian, "Robust delay-distribution-dependent stability of discrete-time stochastic neural networks with time-varying delay," Neurocomputing, vol. 72, no. 4-6, pp. 1265-1273, 2009.

[17] D. Yue, Y. Zhang, E. Tian, and C. Peng, “Delay-distribution-dependent exponential stability criteria for discrete-time recurrent neural networks with stochastic delay," IEEE Transactions on Neural Networks, vol. 19, no. 7, pp. 1299-1306, 2008.

[18] R. Yang, H. Gao, J. Lam, and P. Shi, "New stability criteria for neural networks with distributed and probabilistic delays," Circuits, Systems, and Signal Processing, vol. 28, no. 4, pp. 505-522, 2009.

[19] P. Tiňo, M. Čerňanský, and L. Beňušková, "Markovian architectural bias of recurrent neural networks," IEEE Transactions on Neural Networks, vol. 15, no. 1, pp. 6-15, 2004.

[20] Z. Wang, Y. Liu, L. Yu, and X. Liu, "Exponential stability of delayed recurrent neural networks with Markovian jumping parameters," Physics Letters A, vol. 356, no. 4-5, pp. 346-352, 2006.

[21] H. Huang, D. W. C. Ho, and Y. Qu, "Robust stability of stochastic delayed additive neural networks with Markovian switching," Neural Networks, vol. 20, no. 7, pp. 799-809, 2007.

[22] Y. Liu, Z. Wang, and X. Liu, "On delay-dependent robust exponential stability of stochastic neural networks with mixed time delays and Markovian switching," Nonlinear Dynamics, vol. 54, no. 3, pp. 199-212, 2008.

[23] Y. Shen and J. Wang, "Almost sure exponential stability of recurrent neural networks with Markovian switching," IEEE Transactions on Neural Networks, vol. 20, no. 5, pp. 840-855, 2009.

[24] J. J. Hopfield, "Neural networks and physical systems with emergent collective computational abilities," Proceedings of the National Academy of Sciences of the United States of America, vol. 79, no. 8, pp. 2554-2558, 1982.

[25] K. Hirasawa, S. Mabu, and J. Hu, "Propagation and control of stochastic signals through universal learning networks," Neural Networks, vol. 19, no. 4, pp. 487-499, 2006.

[26] I. Kolmanovsky and T. L. Maizenberg, "Mean-square stability of nonlinear systems with timevarying, random delay," Stochastic Analysis and Applications, vol. 19, no. 2, pp. 279-293, 2001. 
[27] V. B. Kolmanovskii, T. L. Maizenberg, and J.-P. Richard, “Mean square stability of difference equations with a stochastic delay," Nonlinear Analysis: Theory, Methods EApplications, vol. 52, no. 3, pp. 795-804, 2003.

[28] Y. Ji and H. J. Chizeck, "Controllability, stabilizability, and continuous-time Markovian jump linear quadratic control," IEEE Transactions on Automatic Control, vol. 35, no. 7, pp. 777-788, 1990.

[29] X. Mao and C. Yuan, Stochastic Differential Equations with Markovian Switching, Imperial College Press, London, UK, 2006.

[30] E. Zhu, Stability of several classes of stochastic neural network models with Markovian switching, Doctoral dissertation, Central South University, 2007.

[31] S. Boyd, L. EI Ghaoui, E. Feron, and V. Balakrishnan, Linear Matrix Inequalities in System and Control Theory, SIAM, Philadelphia, Pa, USA, 1994. 